\title{
Synthesis, Characterization, and Antibacterial Applications of Novel Copolymeric Silver Nanocomposite Hydrogels
}

\author{
Yonghyun Kim, ${ }^{\dagger}$ V. Ramesh Babu, ${ }^{\dagger}$ Daniel T. Thangadurai,${ }^{\dagger}$ K.S.V. krishna Rao, ${ }^{\dagger, t}$ Hyeongrae Cha, ${ }^{\dagger}$ \\ Changdae Kim, ${ }^{\S}$ Woohong Joo, ${ }^{\#}$ and Yong-III Lee ${ }^{\dagger, *}$
}

\author{
${ }^{\dagger}$ Department of Chemistry, Changwon National University, Changwon 641-773, Korea. *E-mail: yilee@changwon.ac.kr \\ ${ }^{\ddagger}$ Department of Chemistry, Yogi Vemana University, Kadapa 516003, India \\ ${ }^{\S}$ Department of Physics, Mokpo National University, Muan 534-729, Korea \\ \# Department of Biology, Changwon National University, Changwon 641-773, Korea \\ Received June 21, 2010, Accepted December 6, 2010
}

\begin{abstract}
Copolymeric silver nanocomposite hydrogels were synthesized by using acryloyl phenylalanine (APA), $N^{\prime}$-isopropylacrylamide (NIPAM) and crosslinked by $N, N$-methylene bisacrylamide (MBA) via radical redox polymerization. Present study allows entrapping silver nanoparticles into hydrogel networks. UV-visible spectroscopy and X-ray diffraction (XRD) studies confirmed the formation of silver nanoparticles in hydrogel matrix. $11 \%$ of weight loss difference between hydrogel and silver nanocomposite hydrogel is clearly indicates the formation and silver nanoparticles by thermo-gravimetrical analysis. The order of swelling capacity values of hydrogels and silver nanocmposite hydrogels were found to be in the order of placebo copolymeric hydrogel $>$ Ag-copolymeric silver nanocomposite hydrogels. The particle size of silver nanoparticles was analysed and are in the range of $5-10 \mathrm{~nm}$ which has been confirmed by transmission electron microscopy (TEM) as well as particle size analysis. The silver nanocomposite hydrogel has shown very good antibacterial activity on gram-positive and gram-negative bacteriocides.
\end{abstract}

Key Words: Acryloylphenylalanine, Silver nanocomposite hydrogels, Silver nanoparticles, Swelling, Antibacterial study

\section{Introduction}

Hydrogels are the three-dimensional, hydrophilic and partially soluble polymeric networks, which are capable of imbibing large amounts of water or biological fluids. ${ }^{1-4}$ Since, the introduction of hydrogels as novel materials possibly suitable for a broad spectrum of biomedical applications, their research has become a fast-developing and exciting research field. ${ }^{5-7}$ Over past decade, a number of applications involving stimuli-sensitive hydrogels have arisen tremendously due to the great potential for intelligent materials as matrices, actuators and transducers. ${ }^{8-11}$ Owing to the fascinating properties of the stimuli-sensitive hydrogels, which can sense the environmental changes and induce structural changes by themselves, it is certain that they offer many future applications such as suitable materials for the design of intelligent biomaterials and self-regulated drug delivery systems.

The application of nanoscale materials and structures ranging from 1 to $100 \mathrm{~nm}$ is an emerging area in nanoscience and nanotechnology. Nanomaterials may provide solutions to environmental challenges in the areas of solar energy conversion, catalysis, medicine and water treatment. ${ }^{12,13}$ This increasing in the demand must be accompanied by "green" synthesis methods. In global efforts to reduce generated hazardous waste, "green" chemistry and chemical processes are progressively integrating with modern technical developments in science and industry. Implementation of these sustainable processes should adopt the 12 fundamental principles of green chemistry. ${ }^{14-18}$ These principles are geared to guide in minimizing the use of unsafe products and maximizing the efficiency of chemical processes.
Hence, any synthetic route or chemical process should address these principles by using environmentally nontoxic chemicals. ${ }^{14}$

Recent trends demonstrate that the macroscopic gels are becoming most promising as templates/nanoreactors for in situ synthesis of smaller size nanoparticles and this strategy has brought up a new concept in hybrid or composite systems in chemistry and engineering science. ${ }^{19-21}$ A promising antimicrobial coating of Poly (2-hydroxylethyl acrylate) (PHEA) and Poly (ethylenimine) (PEI) networks loaded with Ag nanoparticles, modified with Poly(ehyleneglycol) (PEG) was reported for biomedical and daily-life applications. ${ }^{22}$ Noteworthy, the hydrogel template methodology is that one can control the size and morphology of the nanoparticles by varying the amount of monomer, cross-linker, and functionality of gel networks. ${ }^{23-25}$ Similarly, microgel formulations based on $N^{\prime}$-isopropylacrylamide (NIPAM) in conjunction with nanoparticles are employed for electroanalytical applications. ${ }^{26}$ Ferro-nanohydrogel composites in which magnetic nanoparticles are entrapped inside the hydrogel have been studied recently. ${ }^{27}$ Gels constructed with methyl methacrylate (MMA) and ethylene glycol dimethacrylate (EGDMA) have been employed to control the size and morphology of the palladium nanoparticles. A study by Saravanan et al. ${ }^{28}$ has shown that Poly(acrylamide) hydrogels are effective for the production of nano-sized silver particles ( $\sim \mathrm{nm})$. The combination of metal nanoparticles (silver) with smart polymeric architectures seems to be a promising route to the design of novel materials. Thus herein, we explored the feasibility of precisely producing silver nanoparticles in hydrogel networks and demonstrated their antibacterial activity. 


\section{Experiments and Methods}

Materials. $N^{\prime}$-Isopropylacrylamide (NIPAM), $N, N^{\prime}$-methylenebisacrylamide (MBA), ammonium persulfate (APS) were purchased from Aldrich, USA. $N^{\prime}, N^{\prime}, N^{\prime}, N^{\prime}$-tetramethylethylenediamine (TEMED) was purchased from Alfa Aesar A Johnson Matthey Company. Sodium borohydride $\left(\mathrm{NaBH}_{4}\right)$ was purchased from Acros organics. Silver nitrate $\left(\mathrm{AgNO}_{3}\right)$, L-Phenylalanine and 2,6-di-tert-butyl- $p$-cresol are purchased from SigmaAldrich, USA.

\section{Methods.}

Synthesis of $\boldsymbol{N}$-Acryloyl-L-phenylalanine (APA): $N$-Acryloyl-L-phenylalanine (APA) was synthesized by the procedure given in prior art. ${ }^{29}$ APA was obtained from the usual synthetic route to introduce the vinyl group in the monomers. Aqueous solutions of L-phenylalanine ( $54.5 \mathrm{~g}, 0.33 \mathrm{~mol})$, sodium hydroxide (26.7 g, $0.67 \mathrm{~mol})$ and 2,6-di-tert-butyl-p-cresol (0.10 g) were added drop wise into an acryloyl chloride (29.3 g, 0.31 mol) solution over a $30 \mathrm{~min}$ period at $0{ }^{\circ} \mathrm{C}$. After the addition, the temperature of the reaction medium was raised to room temperature and allowed stirring for a period of $1 \mathrm{hr}$. The whole mixture was acidified to $\mathrm{pH} 2$ with addition of conc. $\mathrm{HCl}(28 \mathrm{~mL})$. A voluminous white product was separated and filtered with suction and purified by recrystallization in water. Reaction mechanism of synthesis of APA has given in Scheme 1.

Synthesis of Poly (NIPAM-co-APA) Hydrogels: NIPAM $(1 \mathrm{~g})$, APA $(180 \mathrm{mg})$ and MBA $(10 \mathrm{mg} / \mathrm{mL})$ were dissolved in double distilled water at room temperature (APA dissolved in $\mathrm{NaOH}$ solution). To this monomer solution, initiator APS and accelerator TEMED were added to allow to polymerize for 24 $\mathrm{h}$ at room temperature. The synthesized copolymeric hydrogels were taken out and immersed in distilled water for 3 days at room temperature by changing the water every $12 \mathrm{~h}$ to remove the ex-

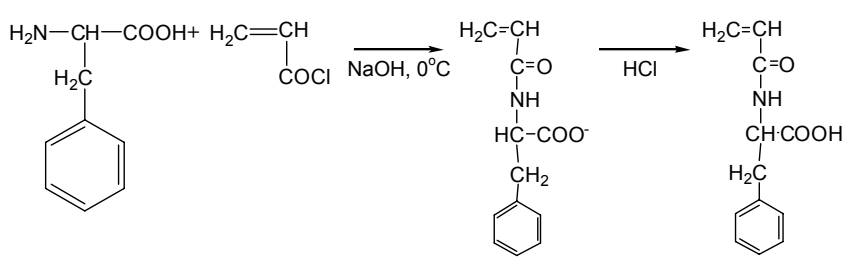

L-Phenylalanine Acryloyl Chloride N-Acryloyl-L-Phenylalanine

Scheme 1. Reaction mechanism and structure of $N$-Acryloyl-L-phenylalanine (APA)

Table 1. Copolymeric hydrogel formulation composition

\begin{tabular}{lcccccc}
\hline \multirow{2}{*}{$\begin{array}{c}\text { Sample } \\
\text { code }\end{array}$} & $\begin{array}{c}\text { NIPAM } \\
(\mathrm{mmol})\end{array}$ & $\begin{array}{c}\text { APA } \\
(\mathrm{mmol})\end{array}$ & $\begin{array}{c}\mathrm{NaOH} \\
(\mathrm{mmol})\end{array}$ & $\begin{array}{c}\text { MBA } \\
(1 \mathrm{wt} \%)\end{array}$ & $\begin{array}{c}\text { APS } \\
(1 \mathrm{wt})\end{array}$ & $\begin{array}{c}\text { TEMED } \\
(\mu \mathrm{L})\end{array}$ \\
\hline APA 5 & 9 & 5 & 5 & $1 \mathrm{~mL}$ & $1 \mathrm{~mL}$ & 50 \\
APA 10 & 9 & 10 & 10 & $1 \mathrm{~mL}$ & $1 \mathrm{~mL}$ & 50 \\
APA 15 & 9 & 15 & 15 & $1 \mathrm{~mL}$ & $1 \mathrm{~mL}$ & 50 \\
APA 20 & 9 & 20 & 20 & $1 \mathrm{~mL}$ & $1 \mathrm{~mL}$ & 50 \\
APA 10-1 & 9 & 10 & 10 & $0.5 \mathrm{~mL}$ & $1 \mathrm{~mL}$ & 50 \\
APA 10-2 & 9 & 10 & 10 & $2 \mathrm{~mL}$ & $1 \mathrm{~mL}$ & 50
\end{tabular}

cess of unreacted monomers and oligomers. During this period, distilled water was refreshed every several hours in order to leach out the residue. The copolymeric hydrogels were prepared by varying the ratios of monomers and crosslinking agent displayed in Table 1.

The loading of silver nanoparticles into the hydrogel networks has been performed by following procedure. Accurately weighed dried copolymeric hydrogels were swelled in double distilled water for 3 days. These swollen copolymeric hydrogels were then transferred into another beaker containing $50 \mathrm{~mL}$ of aqueous $5 \mathrm{mM} \mathrm{AgNO}_{3}$ solution, then allowed equilibrating the hydrogel for $24 \mathrm{~h}$ to absorb the silver salt. During this process, most of the silver ions were exchanged from solution to copolymeric hydrogel and metal ions were occupied in free-network spaces of hydrogel. These silver salt absorbed hydrogels were finally transferred into a beaker containing $50 \mathrm{~mL}$ of $10 \mathrm{mM}$ $\mathrm{NaBH}_{4}$ aqueous solution and allowed for $2 \mathrm{~h}$ to reduce the silver ions into silver nanoparticles. After reduction of silver ions, the hydrogel was turned into brown color, which confirms the formation of silver nanoparticles in copolymeric hydrogel matrix. The formation of silver nanoparticles in hydrogel matrix was given in Scheme 2.

Swelling Studies. Fully dried copolymeric hydrogels were weighed and equilibrated in distilled water at $37^{\circ} \mathrm{C}$ for 3 days. These hydrogels were treated first with $\mathrm{AgNO}_{3}$ then, subsequently with $\mathrm{NaBH}_{4}$ solutions as explained in the experimental section. The equilibrium swelling capacity or swelling ratio $(Q)$ of the hydrogel was calculated employing the following equation (1).

$$
Q=W_{\mathrm{e}} / W_{\mathrm{d}}
$$

Where, $W_{\mathrm{e}}$ is the weight of water in the swollen hydrogel and $W_{\mathrm{d}}$ is the dry weight of the copolymeric hydrogel. The swelling studies were repeatedly performed by thrice with $5 \%$ standard deviation.

Antibacterial Activity Studies. Antibacterial activity screen studies of placebo copolymeric hydrogel and its silver nanocomposite hydrogel were carried out by paper disc method. A quantity of $5 \mathrm{~mL}$ of Nutrient agar (NA) medium $(\mathrm{pH}=6.8)$ was poured into the sterilized plates and allowed to solidify. The plates were inoculated with spore suspensions of three gram-positive ( $M i$ crococcus luteus, Bacillus subtilis and Staphylococcus aureus) and three gram-negative (Proteus vulgaris, Proteus mirabilis and Escherichia coli) bacteria and by using a sterilized cork borer, paper discs (6 mm diameter) were dug inside the culture plates.

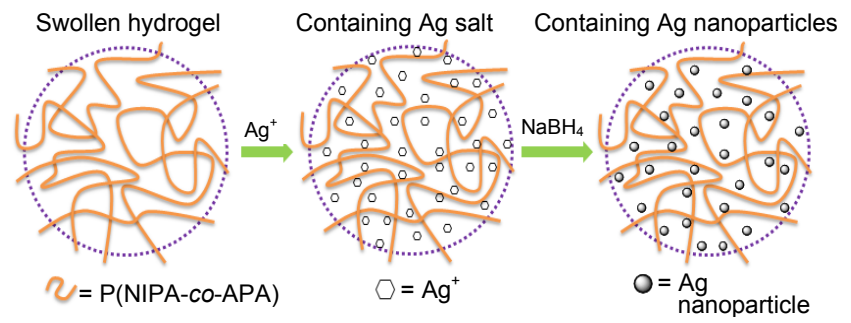

Scheme 2. Schematic illustration of the formation of silver nanoparticles in hydrogel networks 
$100 \mu \mathrm{L}$ of bacteria was used for test. Different concentrations $(1,3$ and $5 \mathrm{mg} / \mathrm{mL})$ of test hydrogel solutions were prepared in distilled water and $30 \mu \mathrm{L}$ of test solution was added to the discs. The plates were incubated at $37^{\circ} \mathrm{C}$ for $24 \mathrm{~h}$. The inhibition zone appeared around the disc was measured and recorded as the antibacterial effect of silver nanoparticles. The antibacterial test was repeated five times to get the reproducibility. To calculate the antibacterial activity of these newly prepared silver-hydrogels statistically, we used the following standard equation (1) to measure the inhibition zone.

$$
\bar{x} \pm \frac{t s}{\sqrt{n}}
$$

Where, $n$ is the number of observations, $\mathrm{s}$ is the measured standard deviation, $\bar{x}$ is the measured mean value of the inhibition zone, and $t$ is Student's $t(t=2.776$ at $95 \%$ confidence level).

Characterization. UV-visible spectra's of copolymeric hydrogel and silver nanocomposites copolymeric hydrogels (10 mg in $1 \mathrm{~mL}$ of distilled water) were obtained using Agilent $8453 \mathrm{UV}$ spectrophotometer. X-ray diffraction measurements were recorded by X'pert MPD 3040 to know the crystallanity of the hydrogel. Thermal properties of copolymeric hydrogels were evaluated by using TA 5000/SDT 2960 DSC Q10 thermal system (Zurich, Switzerland) at a heating rate of $10{ }^{\circ} \mathrm{C} / \mathrm{min}$ under nitrogen atmosphere (flow rate $10 \mathrm{~mL} / \mathrm{min}$ ). Particle sizes of silver nanoparticles are examined by using zetasizer, Malven instruments. The dry hydrogel and silver-nanocomposites hydrogel were coated with a thin layer of palladium gold alloy, and then studied for morphological variations by using a MIRA LMH, H.S scanning electron microscope (SEM). Transmission electron microscopy (TEM) images for hydrogel-silver nanocomposites were recorded using a JEM 2100F transmission electron microscope operating at an acceleration voltage of 15 $\mathrm{kV}$. Samples for TEM were prepared by dropping $10-20 \mu \mathrm{L}$ of finely grinded hydrogel solution on a copper grid and dried at room temperature.

\section{Results and Discussion}

Swelling Studies. Figure 1 shows the influence of various amounts of APA and MBA on swelling characteristics of the hydrogels, silver nanocomposite hydrogels. Authors observed a considerable variation in swelling capacity of the copolymeric hydrogels. The order of swelling capacity values of placebo hydrogels and silver nanocomposite hydrogels are as copolymeric hydrogel > copolymeric silver nanocomposite hydrogels. The electrostatically neutral gels synthesized from ( $N^{\prime}$-isopropylacrylamide) were responsible for a moderate decrement of the swelling behavior of silver nanocomposites. The swelling behaviour of hydrogels has differed from varying the amount of crosslinking agent and APA. As the amount of crosslinking agent increases, the swelling has been decreased due to the high crosslink density with tightening of the polymeric chains which will become rigid structure of the polymer. In case of variation of APA, as the content of APA increases, the swelling
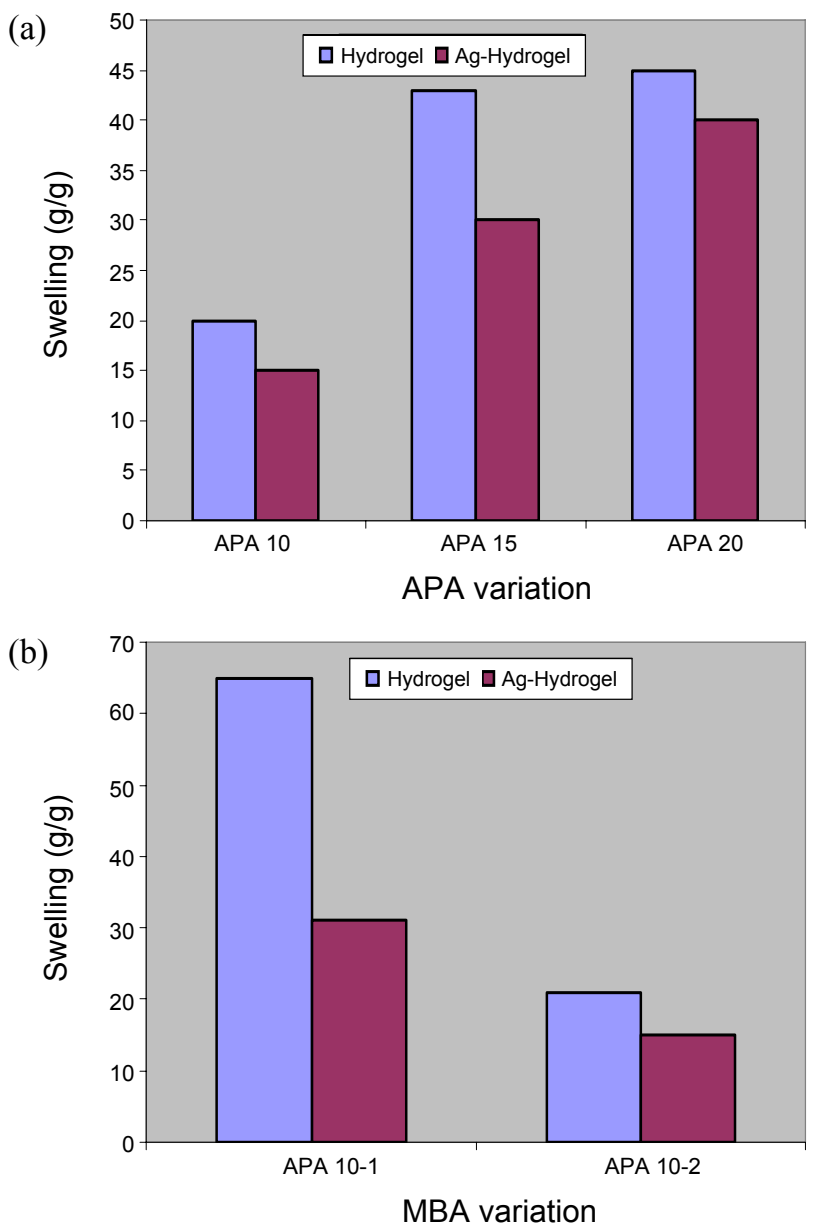

Figure 1. Swelling studies of hydrogel and silver nanocomposite hydrogel with APA (A) and MBA (B).

of the hydrogels increases. This is because of the improving the hydrophilicity of the hydrogel.

UV-visible Spectroscopy. The formation of silver nanoparticles in the copolymeric hydrogel networks can be expected in our current strategy because the silver salts loaded in copolymeric hydrogels are readily reduced by $\mathrm{NaBH}_{4}$, which immediately turn into brown color. It represented that the silver nanoparticles were entrapped inside the polymeric networks. The existences of silver nanoparticles in the gel networks were tested by using UV-visible spectral analysis. Figure 2 illustrates the absorption spectra for copolymeric hydrogel-silver nanocomposites in the range of $300-600 \mathrm{~nm}$. A significant improvement in the absorption peak $\left(\lambda_{\max }=410 \mathrm{~nm}\right)$ was observed for silvercopolymeric hydrogels due to the surface plasmon resonance (SPR) effect. ${ }^{30}$ However, there is no peak around $410 \mathrm{~nm}$ was observed in pure copolymeric hydrogel. This spectral analysis confirmed the formation of silver nanoparticles in the copolymeric hydrogels.

X-ray Diffraction. The X-ray diffraction patterns of placebo copolymeric hydrogel and copolymeric-silver nanocomposite hydrogel were demonstrated in figure 3 . The diffractogram of copolymeric hydrogel-silver nanocomposites are assigned to diffractions at $2 \theta$ values of about $38^{\circ}, 44^{\circ}, 65^{\circ}$ and $79^{\circ}$ plane of face centered cubic (fcc) structure of silver nanoparticles. Sharp 
(a)

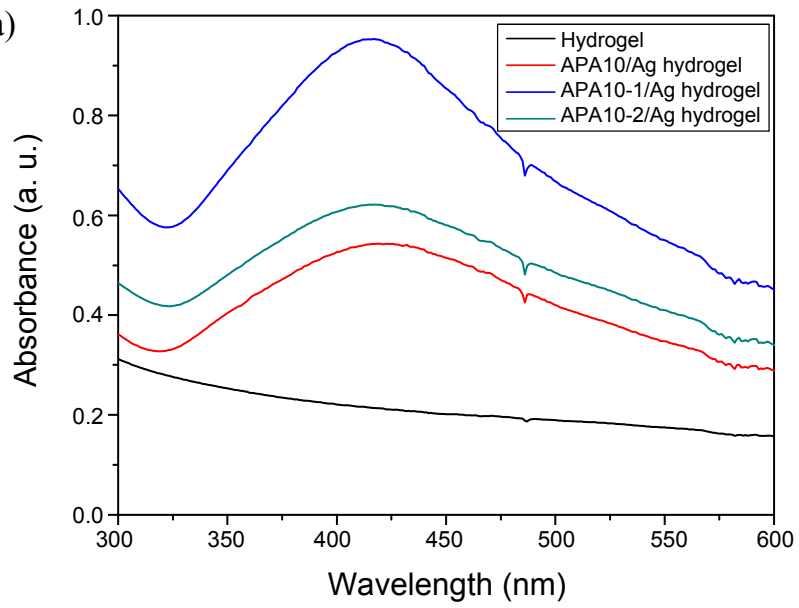

(b)

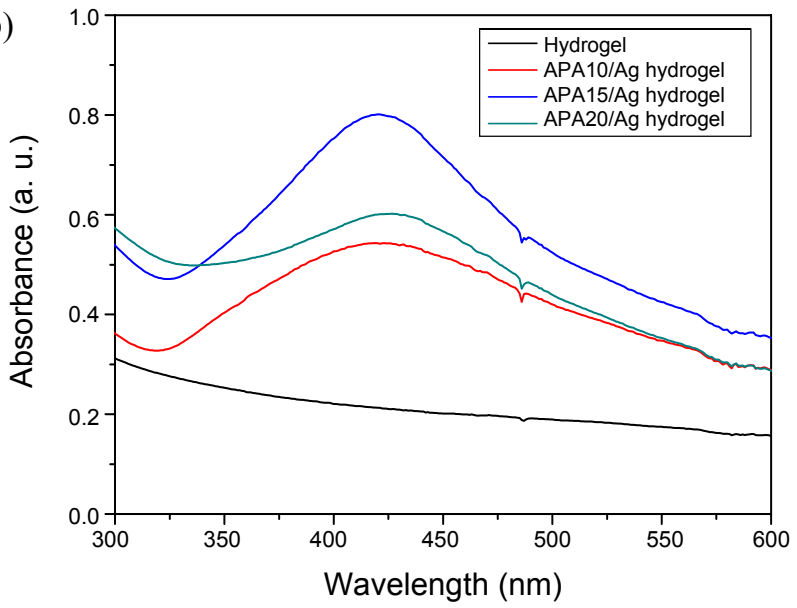

Figure 2. UV-visible spectroscopy of hydrogel and silver nanoparticle hydrogel.

and intense peaks represent the highly crystalline silver nanostructures formed in copolymeric nanocomposites. In pure copolymeric hydrogel there are no peaks observed because of the amorphous nature of Poly(NIPAM-co-APA) hydrogel.

Particle Size Analysis and Scanning Electron Microscopy Studies. Figure 4 illustrates the particle size distribution of silver nanoparticles in the hydrogel matrix. From particle size analysis, it is confirmed that the particle size of silver nanoparticles are in the range of $5-10 \mathrm{~nm}$ with narrow distribution. The scanning electron micrographs of placebo copolymeric hydrogel and copolymeric-silver nanocomposite hydrogel were shown in figure 5. The placebo hydrogel showed a branches and smooth surface (Figure 5a), whereas silver salt-loaded copolymeric hydrogels showed a shrunken surface throughout the gel (Figures $5 \mathrm{~b}$ and $5 \mathrm{c}$ ). However, there is a pinpoint variation in the case of silver nanoparticles formed in the gel networks. Figures $5 \mathrm{~b}$ and $5 \mathrm{c}$ shows the formation of defined nanostructures in the hydrogel networks. This clearly indicates the formation of silver nanoparticles. It is quite common that the control or alignment of nanoparticles can be achieved by modifying the hydrogel network architectures. ${ }^{24}$

Transmisson Electron Microscopy Studies. Nanostructures of the obtained silver nanoparticles are imaged by TEM and illustrated in figure 6 . The TEM image has shown a highly uni-

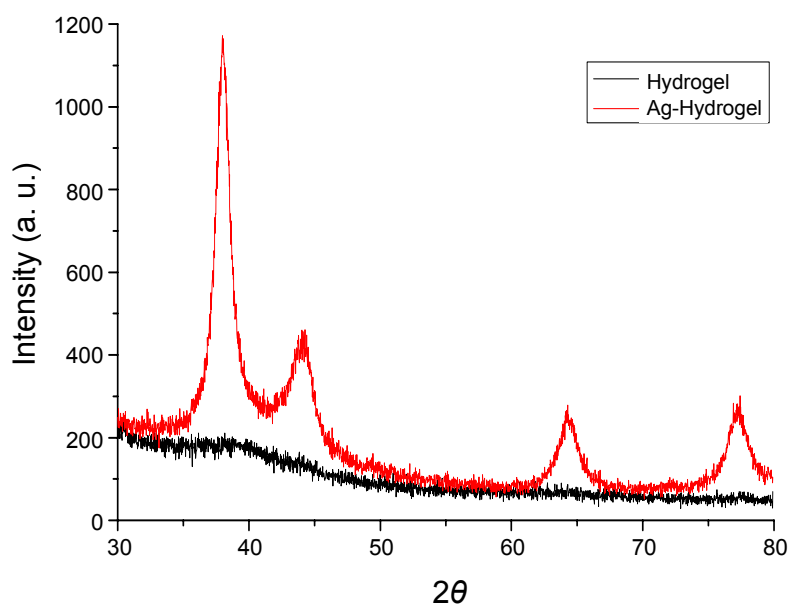

Figure 3. XRD patterns of hydrogel and silver nanoparticle hydrogel

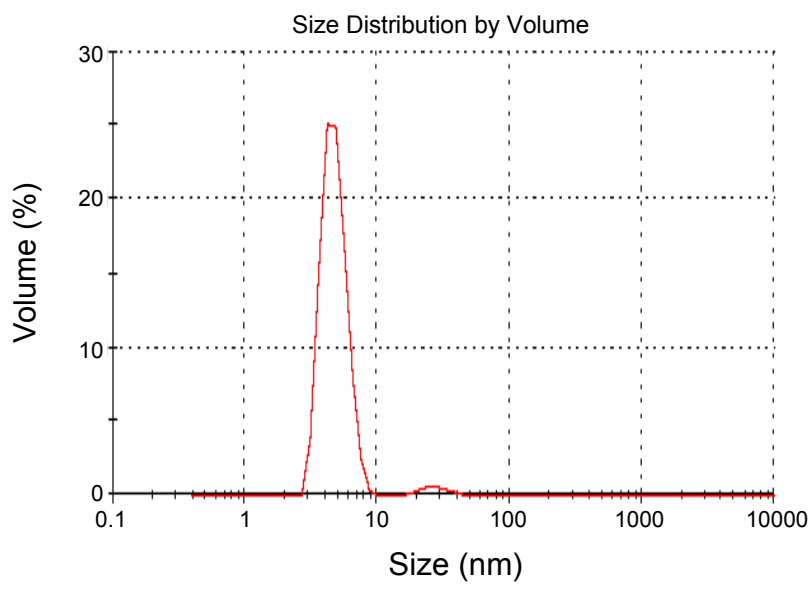

Figure 4. Particle Size analysis of silver nanoparticles.

form distribution of silver nanoparticles. It is clear that the $\mathrm{Ag}$ particles exhibit obvious no difference in size and morphology, due to $\mathrm{Ag}^{+}$ions were loaded at equilibrium swelling of hydrogel before reduction. The Ag particles were small, regular, when $\mathrm{Ag}^{+}$ions were loaded at the equilibrium swelling ratio of hydrogel. It is confirmed that the silver nanoparticles formed in the cross-linked networks are spherical and highly dispersed with $5-10 \mathrm{~nm}$ in size.

Thermal Gravimetrical Analysis. The silver nanocomposite copolymeric hydrogels were characterized by gravimetrical thermo analysis to determine percentage of weight loss of hydrogel as well as silver nanocomposite copolymeric hydrogel matrix. Figure 7 shows the percentage decomposition of hydrogel and silver nanocomposite hydrogel. The hydrogel has followed two decomposition steps and $90 \%$ degradation of the hydrogel chains occurred below $410^{\circ} \mathrm{C}$. However, it is noted as two degradation steps with only $80 \%$ weight loss were occurred even at $900{ }^{\circ} \mathrm{C}$ in the case of silver nanocomposite hydrogel. The difference in decomposition between the hydrogel and silver nanocomposite hydrogel is found to be $11 \%$ and it confirms that the presence of silver nanoparticles (weight loss) in the hydrogel. This is fact due to the presence of silver nanoparticles 

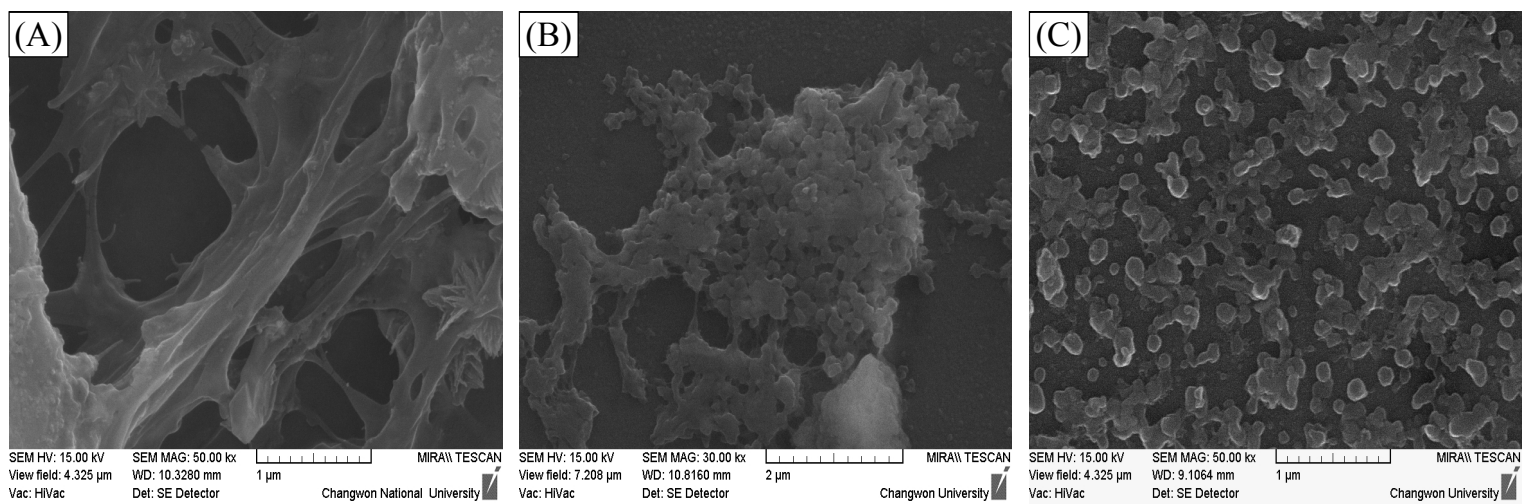

Figure 5. Scanning electron micrographs of pure hydrogel (A) and silver nanoparticle hydrogel (B and C).

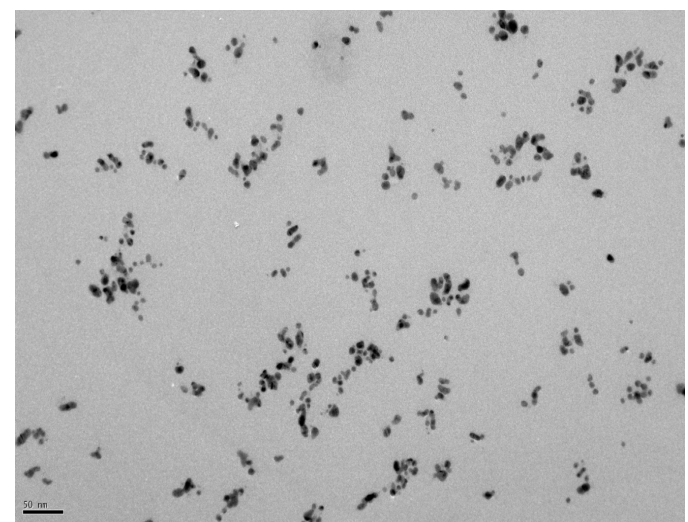

Figure 6. TEM image of silver nanoparticles.

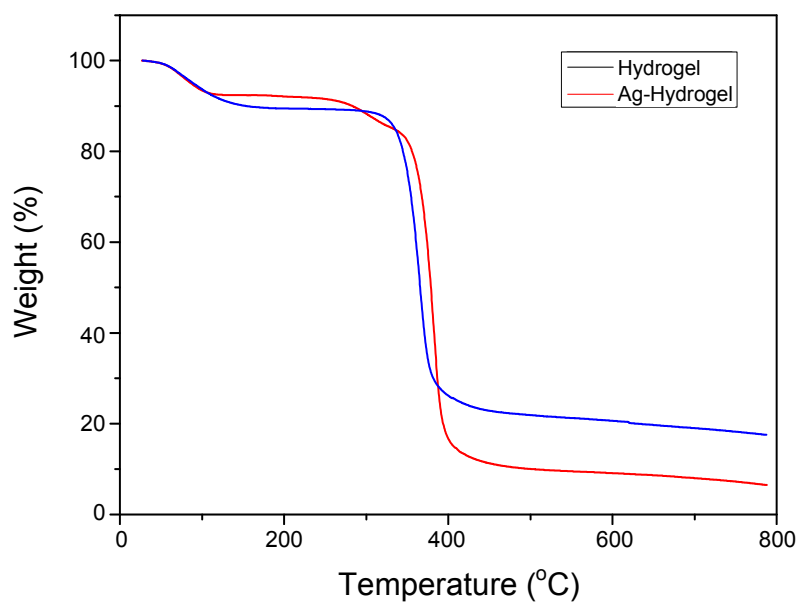

Figure 7. Thermo gravimetrical analysis of hydrogel and silver nanoparticle hydrogel.

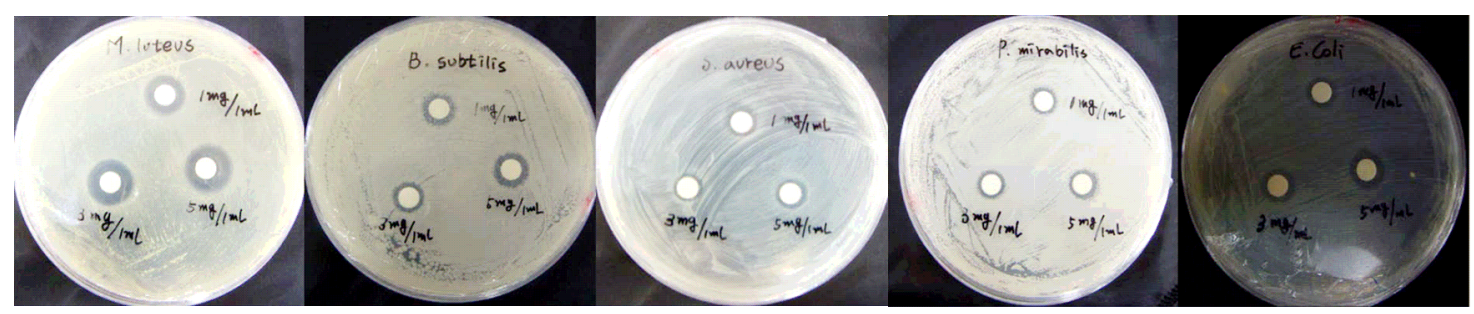

Figure 8. Antibacterial activity picture of silver nanocomposite copolymeric hydrogel's against gram-positive and gram-negative bacteriocides.

Table 2. Antibacterial activity data of silver nanocomposite copolymeric hydrogels against gram-positive and gram-negative bacteriocides

\begin{tabular}{|c|c|c|c|c|c|c|}
\hline \multirow{3}{*}{$\begin{array}{l}\text { Concentration of } \\
\text { Ag-nanoparticle } \\
(\mathrm{mg} / \mathrm{mL})\end{array}$} & \multicolumn{3}{|c|}{ Gram-positive } & \multicolumn{3}{|c|}{ Gram-negative } \\
\hline & M. luteus & B. subtilis & S. aureus & P. vulgaris & P. mirabilis & E. coli \\
\hline & \multicolumn{6}{|c|}{ inhibition zone $(\mathrm{mm})^{a}+$ paper disc width $(\mathrm{mm})^{b}$} \\
\hline 1.0 & $11.8 \pm 0.3$ & $8.7 \pm 0.3$ & $9.1 \pm 0.2$ & $6.8 \pm 0.1$ & $8.7 \pm 0.3$ & $9.3 \pm 0.1$ \\
\hline 3.0 & $12.7 \pm 0.3$ & $9.3 \pm 0.3$ & $9.2 \pm 0.3$ & $8.2 \pm 0.2$ & $9.0 \pm 0.2$ & $9.5 \pm 0.2$ \\
\hline 5.0 & $14.8 \pm 0.2$ & $10.4 \pm 0.2$ & $9.6 \pm 0.2$ & $10.8 \pm 0.3$ & $9.3 \pm 0.3$ & $10.6 \pm 0.4$ \\
\hline
\end{tabular}

$\bar{x}_{\bar{x}} \pm \frac{t s}{\sqrt{n}}, n=5$ at $95 \%$ confidence level, ${ }^{b}$ Paper disc width of $6 \mathrm{~mm}$. 
in hydrogel. As the percentage of metallic nanoparticles increases, difference in weight will be more. In present invention, silver nanoparticles play a role in degradation of hydrogel, which restricts the percentage of weight loss.

Antibacterial Studies. The in vitro antibacterial screening of copolymeric hydrogel and its silver analogues has been carried out against three gram positive and three gram negative bacteriocides. The results suggest that the silver nanocomposite copolymeric hydrogel showed more toxic effect than pure copolymeric hydrogel under similar conditions (Table 2). The possible mode of increased toxicity of silver nanocomposite copolymeric hydrogel may be due to silver nanoparticles coming out easily and could interact with lipid layer of cell membrane. ${ }^{24}$ From the results, we revealed that our silver nanocomposite copolymeric hydrogel showed better activity towards gram-positive bacteria than gram-negative. Figure 8 confirms both antibacterial activity and concentrations are directly proportional, i.e. normally, the biocidal activity increases with increase of concentration.

\section{Conclusions}

Copolymeric silver nanocomposite hydrogels were successfully synthesized free radical polymerization and thereby reduction of silver ions in to silver nanoparticles. The developed silver nanoparticles are well characterized using different techniques, to conform the formation of silver nanoparticles and its anti bacterial activity on gram-positive and gram-negative bacteriocides. Percentage of swelling varied with varying amounts of monomer and crosslinking agent. Thermal analysis, $\mathrm{X}-\mathrm{Rd}$ data and UV-visible spectra revealed the formation of silver nanoparticles in the hydrogel matrix. SEM, particle size distribution curve and TEM images showed the narrow distribution and spherical shape of silver nanoparticles with size range of $5-10 \mathrm{~nm}$. The newly synthesized copolymeric hydrogel silver nanocomposite showed an excellent antibacterial activity and it can be used as drug.

Acknowledgments. This work was supported by Priority Research Centers Program through the National Research Foundation of Korea (NRF) funded by the Ministry of Education, Science and Technology (NRF 2009-0094066).

\section{References}

1. Elvira, C.; Mano, J. F.; Roman, J. S.; Reis, R. L. Biomaterials 2002, 23, 1955.

2. Kopecek, J. Biomaterials 2007, 8, 5185 .

3. Peppas, N. A.; Bures, P.; Leobandung, W.; Ichikawa, H. Eur. J. Pharm. Biopharm. 2000, 50, 27.

4. Wichterle, O.; Lim, D. Nature 1960, 185, 117

5. Hoffman, A. S. Adva Drug Del Rev.1960, 43, 3

6. Van Tomme, S. R.; Storm, G.; Hennink, W. E. Int J Pharma. 2008 , 355,1 .

7. Mahkam, M.; Poorgholy, N.; Vakhshouri, L. Macromol. Res. 2009, 17,709 .

8. Ferreira, L.; Vidal, M. M.; Gil, M. H. Int J Pharma. 2000, 194, 169.

9. Lutolf, M. P.; Lauer-Fields, J. L.; Schmoekel, H. G.; Metters, A. T.; Weber, F. E.; Fields, G. B. Proc. Nat. Acad. Sci. 2003, 100, 5413.

10. Shimoboji, T.; Larenas, E.; Fowler, T.; Kulkarni, S.; Hoffman, A. S.; Stayton, P. S. Proc. Nat. Acad. Sci. 2002, 99, 16592.

11. Szepes, A.; Makai, Z.; Blümer, C.; Mader, P. K., Jr.; Szabo-Revesz, P. Carbohydrate Polymers 2008, 72, 571 .

12. Dahl, J.; Maddux, B. L.; Hutchison, J. E. Chem. Rev. 2007, 107, 2228

13. Hutchison, J. E. Greener Nanoscience 2008, 2, 395 .

14. Anstas, P. T.; Warner, J. C. Green Chemistry; Theory and Practice; Oxford University Press Inc.: New York, 1998.

15. De Simone, J. M. Science. 2002, 297, 799.

16. Cross, R. A.; Kalra, B. Science 2002, 297, 803.

17. Poliakoff, M.; Anastas, T. A. Nature 2000, 413, 257

18. Raveendran, P.; Fu, J.; Wallen, S. L. J. Am. Chem. Soc. 2003, 125 , 13940.

19. Murali Mohan, Y.; Premkumar, T.; Lee, K. J.; Geckeler, K. E. Macromol Rapid Commun. 2006, 27, 1346.

20. Wang, C.; Flynn, N. T.; Langer, R. Adv. Mater. 2004, 16, 1074.

21. Park, M. Y.; Lim, S.; Lee, S. W.; Park, S. E. Macromol. Res. 2009, 17,307

22. Zhao, X.; Ding, X.; Deng, Z.; Zheng, Z.; Peng, Y.; Long, X. Macromol Rapid Commun. 2005, 26, 1784.

23. Lu, Y.; Spyra, P.; Mei, Y.; Ballauff, M.; Pich, A. Macromol. Chem. Phys. 2007, 208, 254.

24. Murali Mohan, Y.; Lee, K. J.; Premkumar, T.; Geckeler, K. E. Polymer 2007, 48, 158.

25. Wang, C.; Flynn, N. T.; Langer, R. Mater. Res. Soc. Symp. Proc. 2004, R2.2.1, 820 .

26. Kazimierska, E. A.; Ciszokowska, M. Electroanalysis 2005, 17, 1384.

27. Mayer, C. R.; Cabuil, V.; Lalot, T.; Thouvenot, R. Adv. Mater. 2000, 12, 417 .

28. Saravanan, P.; Raju, M. P.; Alam, S. Mater. Chem. Phys. 2007, 103, 278

29. Casolaro, M.; Paccagnini, E.; Mendichi, R.; Ito, Y. Macromolecules 2005, 38, 2460.

30. Acharya, H.; Sung, J.; Shin, H.; Park, S. Y.; Min, B. G.; Park, C. Reactive \& Functional Polymers 2009, 69, 552. 